\title{
Using NEXUS Compliant Debuggers for Real Time Fault Injection on Microprocessors
}

\author{
André Fidalgo, Manuel Gericota, Gustavo Alves \\ Department of Electrical Engineering - ISEP \\ Rua Prof. Bernardino de Almeida 431 \\ P-4200 Porto, PORTUGAL \\ $+351.22 .8340500$ \\ \{anf, mgg, gca\}@isep.ipp.pt
}

\begin{abstract}
As electronic devices get smaller and more complex, dependability assurance is becoming fundamental for many mission critical computer based systems. This paper presents a case study on the possibility of using the on-chip debug infrastructures present in most current microprocessors to execute real time fault injection campaigns. The proposed methodology is based on a debugger customized for fault injection and designed for maximum flexibility, and consists of injecting bit-flip type faults on memory elements without modifying or halting the target application. The debugger design is easily portable and applicable to different architectures, providing a flexible and efficient mechanism for verifying and validating fault tolerant components.
\end{abstract}

\section{Categories and Subject Descriptors}

B.8.1 [Hardware]: Performance and Reliability - Reliability, Testing, and Fault-Tolerance.

\section{General Terms}

Design, Reliability, Experimentation, Standardization.

\section{Keywords}

Fault Injection, Real Time Systems, On Chip Debug

\section{INTRODUCTION}

Today, most safety-critical applications require the use of some type of computer-based device, causing their implantation to grow and expand into new areas like the automotive and biomedical fields. However, as electronic systems increase in complexity and decrease in size their correct operating behavior is becoming harder to guarantee [1]. Circuits are getting more sensitive to noise and to other factors, with the appearance of soft errors becoming a real possibility even for devices used in non-hostile environments, making dependability a necessity for a much broader area of applications. Dependable systems are designed to handle errors that originate from software or hardware faults and

This work is supported by an FCT program under contract POSC/EEAESE/55680/2004

Permission to make digital or hard copies of all or part of this work for personal or classroom use is granted without fee provided that copies are not made or distributed for profit or commercial advantage and that copies bear this notice and the full citation on the first page. To copy otherwise, or republish, to post on servers or to redistribute to lists, requires prior specific permission and/or a fee.

SBCCI'06, August 28-September 1, 2006, Minas Gerais, Brazil.

Copyright 2006 ACM 1-59593-479-0/06/0008...\$5.00.

\author{
José Ferreira \\ Department of Electrical Engineering - FEUP \\ Rua Dr. Roberto Frias, s/n \\ P-4200-465 Porto, PORTUGAL \\ +351.22. 5081400 \\ jmf@fe.up.pt
}

to recover from them, while maintaining acceptable operating conditions. The possibly destructive nature of a failure and the long error latencies impair identifying the cause of failures in field operation and in the normal time that it takes for a failure to occur. To identify and understand potential errors, it is desirable to experiment on an actual device as to better study and improve its dependability. This approach can be applied either on the development phase, where models or prototypes are used, or on the deployment phase, if faults can be deliberately injected in useful time without damaging the equipment. This experimentbased approach requires knowledge of the system architecture and behavior, and especially of the mechanisms implemented to provide tolerance to faults, errors or failures, i.e. the events leading to a service failure on microprocessor based systems [2]. Specific instruments and tools must be used to induce these hazards and monitor their effects and in the case of microprocessor systems, access to the internal resources is of utmost importance. Many of today's microprocessors provide such access through dedicated built-in debug circuitry, often designated as on-chip debug (OCD). The use of these OCD infrastructures for fault injection purposes is an efficient solution for verifying and validating fault tolerant designs. This paper describes recent research on real time fault injection (i.e. without halting application execution) targeting such devices, based on the development and use of a debugger optimized for fault injection. The rest of the paper is organized as follows: the next section gives an overview of fault injection methodologies used on microprocessor systems and previous work on this area; section 3 presents the system used as a case study, the fault injection oriented debugger and some proposals for enhanced fault injection support; section 4 presents the experimental results obtained so far and finally section 5 discusses these results and lays the basis for future work.

\section{FAULT INJECTION METHODOLOGIES 2.1 Overview}

In microprocessor systems, the most common methodology to achieve dependability is the use of fault-tolerant components, both in hardware and software. The correct behavior of such components must be tested and fault injection can be used to (1) identify design or implementation faults, (2) verify \& validate fault tolerance capabilities and (3) estimate how often failures will occur and evaluate the consequences of such failures.

Fault injection is normally structured in campaigns, each being composed of a series of experiments during which the target system runs (a specific application is executed) and a specific fault (or set of faults) is inserted at specific trigger conditions. The target system behavior is monitored and information is recorded 
as comprehensively as necessary and possible, to later understand and evaluate the effects of the inserted fault(s).

Existent microprocessor fault injection techniques are commonly classified in three broad groups, namely (1) simulation based fault injection, (2) software based fault injection (SWIFI), and (3) physical fault injection.

Simulation based fault injection is mostly used in the early phases of a design when the target system exists only in model format. This technique requires a model of the target itself, (normally in some HDL format), the necessary simulation tools to insert faults and adequate processing capabilities to run the simulation [3].

Software based fault injection consists of reproducing at a logical level the errors originated by physical faults using software commands already available on the target device. This allows the injection of errors on all resources accessible by software, like registers, program and data memory, most peripherals and some timers [4]. Physical fault injection is a more realistic approach in the sense that it tries to replicate real world faults. All physical techniques perform an actual fault insertion on the circuit or emulate their immediate consequences (errors) through internal or external action. Access to the circuit elements is usually performed either through specific hardware equipment [5] or using debug and test infrastructures included on the target chip [6]. Physical fault injection may also be performed without a direct connection between the fault injector and the system under test, either through laser [7], heavy-ion radiation or electromagnetic fields [8].

The hardest part of microprocessor fault injection is how to access those internal elements where faults are more probable, generally the memory elements and communication buses, without disturbing the running applications. OCD infrastructures provide access to internal resources in parallel with the target hardware and running software, being an excellent mechanism for modifying register and / or memory values (i.e. insert faults) and subsequently retrieve the data necessary for result analysis.

The OCD facilities implemented by different families of processors share some common characteristics that form a core feature set, which usually includes run-control, breakpoint support and memory and register access. Some devices include more advanced features like watchpoints, program trace and real time debugging capabilities. In general, an OCD is a combination of hardware and software on the microprocessor chip that requires some external hardware to be used, the basic requirement being some kind of communication link between the chip and the host machine. The access to the OCD infrastructure is made through an interface port usually requiring an external debugger in between.

The use of OCD infrastructures for fault injection can overcome some of the limitations present on other approaches. For instance, simulation techniques are often time-consuming and may lead to erroneous results as they are intrinsically dependant on the quality of the available model. SWIFI techniques require modifications to the running code, which in fact modifies the target system, and coverage is limited to the resources accessible by software. Most physical fault injection techniques are expensive and precise control of the instant and location of a fault is often very difficult or even impossible. In most cases, OCD fault injection techniques rely on halting the processor, either by the use of control signals or breakpoints, and subsequently modifying the targeted registers or memory locations to insert the intended faults. When available, program or data trace provide an efficient mean to monitor fault propagation and effects. Recent OCD implementations provide added capabilities like real-time access to memory and online trace data output. These can be effectively reused for real-time fault injection in the sense that it is no longer necessary to halt the target execution to insert faults.

\subsection{Using commercial NEXUS debuggers}

As a technological solution, a major problem with OCD is the lack of a consistent set of capabilities and a standard communications interface across processor architectures. An industry consortium has been working on the establishment of a standard for OCD, which is still on a proposal phase and is formally designated as "IEEE-ISTO 5001, The Nexus 5001 Forum Standard for a Global Embedded Processor Debug Interface" [9]. If widely adopted, it may be possible to employ the same debugger to access the core of multiple processor architectures and to use a similar set of debugging features for all. Additionally, the feature set that this standard proposes for the higher classes of compliance provides a useful set of tools for real time fault injection in the form real time access to memory and on-the-fly program and data trace.

Experimental work has been done in our research group and in the DISCA-UPV [10] to evaluate the possibilities of executing realtime fault injection on a NEXUS compliant microprocessor. The target systems used were based on a Motorola MPC565 CPU [11], which is a commercial 32 bit microcontroller with widespread use on the automotive industry. The OCD infrastructure available on the MPC565 devices is NEXUS Class $2+$ compliant and includes run control, watchpoint and breakpoint support, real time access to memory (RAM only), access to all memory space and registers on DEBUG mode (i.e. execution is halted). Trace support is very flexible, being possible to log program and/or data accesses and start the trace process on specific conditions, similar to those available for breakpoint detection. In our case, the debugger used was an iSystems IC3000 [12] (iTracePro version) and its integrated debugging software Winidea 2005. This software allows direct control of the debugger and the use of scripts (running on the host machine) to automate the debugging tasks. The fault injection environment is presented in Figure 1.

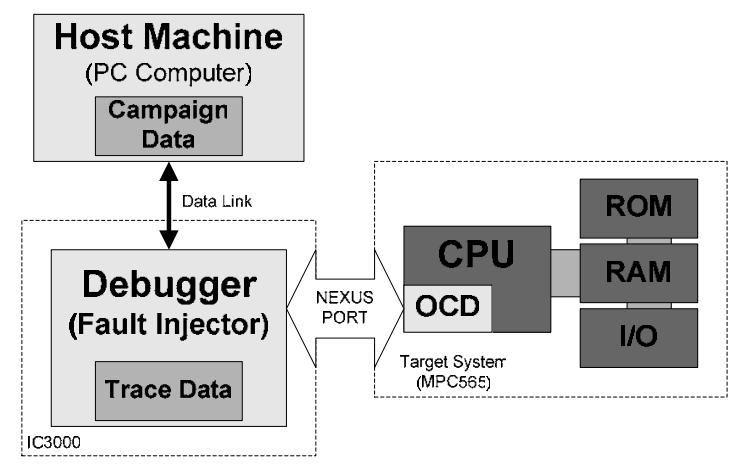

Figure 1. Fault Injection Environment (MPC565)

The fault campaigns were manually generated and translated into Winidea scripts. A typical fault injection operation would require the microprocessor to run until the triggering condition was met, this being signaled to the host machine so that it could instruct a memory access operation (via debugger and OCD) to inject the intended fault. Two triggering options are available as the direct use of a watchpoint signal is not possible on the Winidea environment, namely: (1) injecting the fault after a specific period 
of time, as measured by the host clock or (2) use the start of the trace data recording to trigger the fault injection process.

The actual fault injection consists of reading the target memory cell content, modifying it and then writing the faulty value on the same cell. If the value of the target cell at the fault triggering instant can be determined beforehand then the read operation can be bypassed and the faulty value written immediately.

The obtained results confirmed most of the expected potentialities and simultaneously identified some shortcomings both in fault triggering and performance. It proved possible to insert faults in memory space without affecting the running application and then use the trace information gathered as an effective mean to analyze program flow, before and after the actual fault activation. However, as all NEXUS compliant debuggers currently communicate with the host machine through Ethernet or USB connections, and as the fault campaigns must be run on the host machine, this imposes a bottleneck on the time required for an actual memory access. This fact causes the time interval required for reading a memory cell contents and writing back a modified value to be measured in milliseconds. This delay allows the initial data to be overwritten by the application running on the target system, the magnitude of the problem depending of the running application and memory position targeted, leading to inconclusive results. An additional problem is the triggering of a fault. Even using the trace data without halting the processor the required information is not readily available, as it must reach the host machine before it can be acted upon. This additional delay is also in the range of milliseconds, limiting the practicability of its use for triggering, because once action is taken the original event has long passed and the precise delay can't be accurately determined. Both the described problems are not directly related with the OCD capabilities but rather with the available tools, which lack some features that, not being necessary for debug, would be very useful for fault injection. The probability of the running application overwriting the targeted cell during the fault injection process can be minimized by reducing the writing delay of the fault injection process. The triggering delay problem can be solved by adding reactive behavior to the debugger so that it can perform a write operation on the detection of a specific (1) signal or (2) message from the target system. Both these solutions can be addressed by a debugger with the required capabilities.

\section{CASE STUDY}

\subsection{Target System}

The use of a NEXUS compliant debugger benefits from the useful features defined in this standard and increases the area of immediate applicability of the developed concepts and solutions. As neither the actual compatible CPUs nor the commercial debuggers are easily modifiable, the reported case study requires (1) an alternative microprocessor core where a compliant OCD infrastructure could be implemented and (2) a customized debugger, as specific libraries are required for each target. The OCD and the debugger itself were developed as two distinct VHDL modules, aiming to keep them simple and easily portable to maintain a high level of compatibility with different target architectures. In this way a complete proof-of-concept solution was tested and the requirements for its migration to existent systems (or under development) were evaluated.

The cpugenerator [13] building tool was selected to create the different microprocessor targets. It is publicly available through opencores [14] and allows the automatic creation of $4,8,16$ or 32 bit RISC microprocessor cores, being possible to configure several parameters like bus type, interrupt support and memory configuration. The OCD version implemented on the target system is NEXUS Class 2 compliant and provides some customization features, to be compatible with different CPU configurations with only minor adjustments. It is possible to define the data bus width (input and output) and the internal FIFOs used to store data prior to its decoding or communication. These parameters are very important as they may constrain the capabilities of the OCD in terms of trace and real time access. On the other hand, the use of larger buses can significantly increase the logic overhead imposed by the OCD infrastructure. The target application for testing is a Matrix_addFT program, which is a fault tolerant version of a matrix adder. This was selected as it is simple to debug and also memory intensive. The fault tolerance is achieved by duplicating each arithmetic operation and then comparing the obtained results, with any difference triggering an error detection routine. Although not as powerful as hardware fault tolerance, this solution allows for some degree of dependability without modifications to the hardware, at the cost of memory space and some performance penalty.

The NEXUS standard defines a minimum set of debugging features, the interface port and the communication protocol. The implemented features include all common OCD features plus real time access to memory. The interface with the outside world is made using the AUX port option, which provides two message data buses for OCD data input and output along with independent clock and control signals. Two additional event pins allow halting the processor and provide exact timing for watchpoint / breakpoint signaling. The communication protocol followed the NEXUS standard spec, with all mandatory messages being included and two additional optional messages added for internal register access and OCD configuration.

\subsection{Fault Injection Environment}

The selected fault model is the one used in most common fault scenarios for microprocessor based critical systems [15] and consists of single bit-flip faults in random memory elements at also random moments during the application execution. The actual fault trigger can be any instruction occurrence of the running application, covering the entire execution time. The fault location can be any resource accessible for writing through the OCD, including memory and internal registers. As memory space can be accessed with the target application running, this is the area where the proposed solution presents the highest advantages. Real time access to internal registers would be intrusive, and it is not possible with actual OCD implementations. In this case, the objective is the reduction of the interval during which the execution is halted.

All experiments are structured into fault injection campaigns, each one defining a set of fault injection operations where specific fault coordinates (location $\mathrm{x}$ value) and trigger condition are selected. In each such operation the processor is reset and the application runs from start. Each campaign is generated by an external tool and then described as a script with the necessary messages to be sent to the OCD infrastructure, both for configuration and data collection. Initialization is performed by loading the application into memory and setting up the OCD infrastructure as required by the specific operation. The target memory value at the moment of the injection must be determined beforehand, using either the knowledge of the running application 
code or a prior faultless execution up to the fault triggering instant and then using the OCD to read the relevant memory cell contents. In this manner it is possible to determine the value that should be stored so that a single bit-flip is caused on the target with a single write operation. The fault trigger condition is selected from the executed application code and can be any event that triggers a watchpoint, like an instruction execution or a data access. The normal fault injection scenario consists of the NEXUS compliant target microprocessor, the debugger running the fault injection campaigns and the host machine which is only used for debugger set up (data upload) and posterior analysis (data download). This is represented in Figure 2.

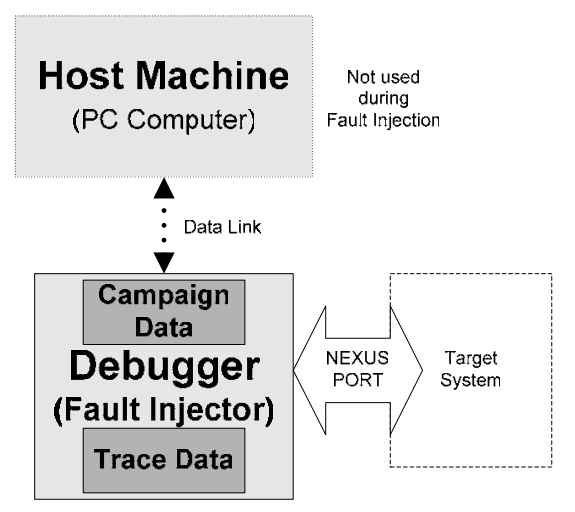

Figure 2. Fault Injection Environment (Case Study)

The main advantage of this fault injection solution is the debugger capability to manage the entire fault injection process. Although the host machine is responsible for downloading the fault campaign data to the debugger and uploading the trace data after the fault campaign execution, the entire fault campaign is executed autonomously by the debugger. Additionally, if the target system is implemented on a FPGA device it is possible to add the debugger (and all relevant fault campaign data) as a module implemented on the same device, with the inherent advantages in terms of performance and cost.

Each fault injection operation consists of loading the debugger input memory with a series of instructions describing the steps required for its execution. After the initial set up is completed, the debugger waits for the triggering condition to be met, which will be signaled by a watchpoint hit signal or by a breakpoint hit message. When either of these events occurs the debugger sends a message to the OCD instructing it to write into the target memory position the intended faulty value. Although the debugger allows an instantaneous reaction, the actual fault insertion requires the transmission and decoding (by the OCD) of at least one complete message (the write command and data). During the entire operation the output memory records the trace messages that are sent by the OCD, to allow a subsequent program flow reconstruction and fault effect analysis. From these messages it is possible to diagnose fault effects, verifying if the fault was acknowledged by the error detection routine, and after the application runs its course it is possible to use the OCD to check if all final results are correct. All set up steps can be done with the target processor running normally, but the fault activation may only take place after this set up is performed. The program trace is not affected and operates normally before, during and after the fault injection process, reacting exactly as if a "real" fault occurred.

\subsection{Debugger}

The debugger allows the execution of the common debugging operations, and was designed to optimize the execution of fault injection operations with emphasis on execution speed. It can be used to control the target system via the OCD, enabling run control and non-intrusive access to most target resources (depending on OCD capabilities). Figure 3 presents its internal structure and main components, namely the debugger core, the two memory banks (input and output) and the NEXUS communication port.

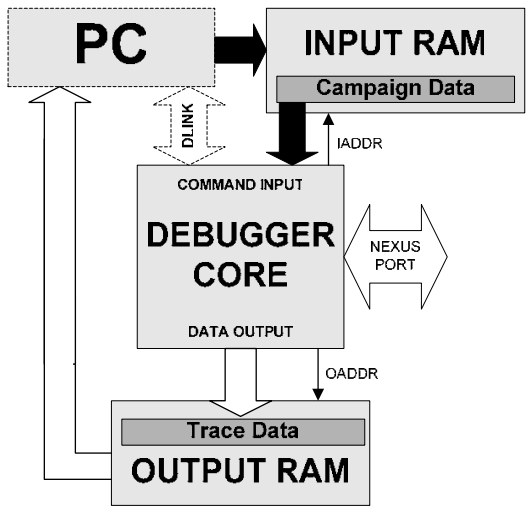

Figure 3. Debugger

The debugger core is a simple processor type device that fetches commands from the input memory, controls execution and manages the data flow and possible error conditions. Table 1 displays a list of available commands and the corresponding parameters.

Table 1 - Debugger Commands and Parameters

\begin{tabular}{|c|c|c|}
\hline COMM & PARAM & DESCRIPTION \\
\hline HALT & None & $\begin{array}{l}\text { Halts target execution and enters } \\
\text { DEBUG mode. }\end{array}$ \\
\hline RUN & None & $\begin{array}{l}\text { Starts or resumes the target } \\
\text { microprocessor execution. }\end{array}$ \\
\hline RESET & None & Resets the target microprocessor. \\
\hline DRESET & None & $\begin{array}{l}\text { Resets the debugger, restarting } \\
\text { command fetch from the initial } \\
\text { input memory position. }\end{array}$ \\
\hline DCONFIG & $<$ code $>$ & $\begin{array}{l}\text { Configures the debugger according } \\
\text { to the }<\text { code }>\text { parameter. }\end{array}$ \\
\hline WAIT & $<$ time $>$ & $\begin{array}{l}\text { Waits for a number of clock cycles } \\
\text { defined by the }<\text { time }>\text { parameter. }\end{array}$ \\
\hline WAITFOR & $\begin{array}{l}<\text { event }> \\
<\text { time }>\end{array}$ & $\begin{array}{l}\text { Waits for a specific message or a } \\
\text { watchpoint hit signal from the } \\
\text { target OCD, during a specific } \\
\text { period of time. The messages can } \\
\text { be any response or trace message. }\end{array}$ \\
\hline READRAM & $<$ address $>$ & $\begin{array}{l}\text { Reads the contents of the memory } \\
\text { cell at the specified address. }\end{array}$ \\
\hline WRITERAM & $\begin{array}{c}<\text { address }> \\
<\text { data }>\end{array}$ & $\begin{array}{l}\text { Writes a byte of data to the } \\
\text { memory cell at a specified address. }\end{array}$ \\
\hline READREG & $<$ address $>$ & $\begin{array}{l}\text { Reads the contents of a register at } \\
\text { the specified address. }\end{array}$ \\
\hline WRITEREG & $\begin{array}{c}<\text { address }> \\
<\text { data }>\end{array}$ & $\begin{array}{l}\text { Writes a byte of data to the register } \\
\text { at the specified address. }\end{array}$ \\
\hline
\end{tabular}


Direct control is possible through specific signals (DLINK) which may replace either the input or output memories (or both) as source of commands and destination of data.

The access to the input memory, for reading purposes, is controlled by the debugger core and executed sequentially. The output memory is used to store data for subsequent program flow analysis. The type of information stored can be selected by configuring the debugger and depends on the task at hand and available memory. The NEXUS port is managed by a communication controller responsible for translating commands into messages to be sent and retrieving the messages received from the OCD. The width of the data buses defines the duration of the transmission required by each message.

\subsection{Performance Improvements}

The fault injection procedure described on the previous subsections was planed with the double objective of improving the performance and maintaining the highest level of compatibility with different target microprocessor architectures. It is possible to improve performance even further by modifying the OCD infrastructure present on the target microprocessor. Two approaches requiring modifications to the OCD were tested, namely (1) the simplification of the communication between the debugger and the OCD and (2) the migration of the reactive behavior to the OCD infrastructure itself. The first approach implies modifications to both the OCD and the debugger and consists of removing the NEXUS interface on both modules and connecting the debugger directly to the OCD internal signals and registers. The advantage is the elimination of the coding and decoding of the NEXUS messages and the inherent delay induced by those steps, however this approach can only be applied either in simulation or using a special version of the target system. The second approach is described in more detail in [16] and consists of adding an extra module to the OCD infrastructure in order to allow it to control part of the fault injection process. In this alternative the debugger and the NEXUS interface are unchanged, the differences being in the sequence of commands used for each fault injection operation as the actual triggering of the fault and memory writing is executed by the enhanced OCD itself.

\section{EXPERIMENTAL RESULTS}

The target system, the debugger and the different memories were designed as VHDL models using the ISE 7.1i development environment [17] and simulated using the Modelsim 6.0a simulation engine. Four different CPU and OCD combinations were used, as summarized in Table 2. The MPC565 is included for comparison purposes, the values representing the best possible configuration. The CPU configurations differ only in terms of bus width. The OCD configurations vary in terms of port width and on the size of the internal message buffers, with MDI being the Message Data In bus and MDO the Message Data Out bus.

Table 2 - Target System Configurations

\begin{tabular}{|l|c|c|c|c|}
\hline Configuration & $\begin{array}{c}\text { BUS } \\
\text { (bits) }\end{array}$ & $\begin{array}{c}\text { CLK } \\
\text { (MHz) }\end{array}$ & $\begin{array}{c}\text { MDI } \\
\text { (bits) }\end{array}$ & $\begin{array}{c}\text { MDO } \\
\text { (bits) }\end{array}$ \\
\hline CPU8a & 8 bits & 100 & 1 bit & 1 bit \\
\hline CPU8b & 8 bits & 100 & 2 bits & 4 bits \\
\hline CPU32a & 32 bits & 25 & 2 bits & 8 bits \\
\hline CPU32b & 32 bits & 25 & 4 bits & 8 bits \\
\hline MPC565 & 32 bits & 40 & 2 bits & 8 bits \\
\hline
\end{tabular}

CPU8a and CPU8b represent the minimal and recommended configurations for 8 bit microprocessors, while CPU32a represents a configuration equivalent to the best available for the MPC565 microprocessor and CPU32b represents an improved configuration for faster memory writing. All configurations include separate ROM and RAM banks on the target system, the first for storing the program code and the later for application data. The fault campaigns were structured as follows:

- Each campaign is loaded into memory and the experiments are executed sequentially with the target CPU being RESET between experiments.

- The instruction address that triggers each fault injection is randomly generated from the actually executed ROM space and the target memory position is randomly selected from the actually used RAM space.

- The OCD is configured once at the beginning of the campaign, with the configuration depending on the fault injection target (memory or registers). Depending on the target the trigger is configured as a watchpoint (fault insertion on-the-fly) or a breakpoint (execution is halted to insert the fault).

- The results are retrieved after all the experiments are complete and their analysis is performed externally with each experiment being diagnosed, to check if the final results are correct and if the fault was detected by the fault tolerance routine.

The simulation of about 100 fault campaigns repeated for each configuration returned the results presented in Table 3 -. In this table, (1) OCD errors represent the fault campaigns that were impossible to terminate due to trace overflow errors, (2) inconclusive results represent experiments that had to be discarded due to multiple bit-flip error insertion (caused by a modification to the target memory during the fault injection process), and (3) fault injection delay represents the time interval between the meeting of the trigger condition and the actual insertion of the faulty value as obtained from the simulation waveforms.

Table 3 - Fault Injection Results

\begin{tabular}{|c|c|c|c|c|c|}
\hline \multicolumn{2}{|c|}{ Configuration } & CPU8a & CPU8b & CPU32a & CPU32b \\
\hline 1 & OCD Errors & $88 \%$ & 0 & 0 & 0 \\
\hline 2 & $\begin{array}{c}\text { Inconclusive } \\
\text { Results }\end{array}$ & 0 & $2 \%$ & $4 \%$ & $3 \%$ \\
\hline 3 & $\begin{array}{c}\text { Fault } \\
\text { Injection }\end{array}$ & 25 & 14 & 24 & 21 \\
\hline
\end{tabular}

Some conclusions, relative to the fault injection process, are possible at this stage:

- It wouldn't be possible to execute the same fault campaigns (on real time) on a system using an MPC565 and a commercial controller as the reaction delay would be to high for this particular application (the total execution time is less than the interval required for injecting a single fault).

- Using configuration CPU8a causes a very high number of OCD trace overflow errors due to the reduced MDO bandwidth, making it impracticable to use this configuration for fault injection.

- When targeting memory in real time, some experiments return inconclusive results because the CPU writes on the memory cell being targeted before the fault is actually inserted. 
- The width of the communication channel between the debugger and the OCD clearly affects the performance of the fault injection process, with the use of larger buses reducing the occurrence of inconclusive results.

- The number of equivalent gates for each module and each target configuration is given by Table 4 .

Table 4 - Area Overhead

\begin{tabular}{|c|c|c|c|c|}
\hline \multirow{2}{*}{ Module } & CPU8a & CPU8b & CPU32a & CPU32b \\
\cline { 2 - 5 } & \multicolumn{4}{|c|}{ \# Equivalent Gates } \\
\hline CPU core & 9166 & 9166 & 53717 & 53717 \\
\hline OCD & 6217 & 6985 & 17601 & 18801 \\
\hline $\begin{array}{c}\text { Debugger } \\
\text { (except RAM) }\end{array}$ & 766 & 766 & 1079 & 1079 \\
\hline
\end{tabular}

From the above values it is possible to confirm that a simple debugger (tasked only with fault injection campaigns management and results storage) requires comparatively little space on a programmable device.

\section{CONCLUSIONS AND FUTURE WORK}

Dependability evaluation efforts sometimes neglect the possibilities of powerful OCD infrastructures present on the target device, even knowing that their use as a mean to execute nonintrusive real-time fault injection campaigns is often the best solution in terms of performance and capabilities. The reasons behind this are sometimes lack of appropriate tools or inadequate documentation. The diversity of methodologies, feature implementation and interface ports is also a downside. Our case study shows that the use of an optimized debugger and an OCD with real time access capabilities allows the execution of fault campaigns on the target memory space with full coverage of the application execution and all resources accessible through the OCD. The possibilities in terms of fault triggering, fault injection delay and fault coverage are dependent on the OCD capabilities. Communication speed is the fundamental factor as the use of larger communications ports allows faster operation and therefore minimizes the risk of the running application interfering with the process. In terms of coverage all resources accessible for reading and writing can be targeted, and real time access is a big advantage. Although some resources (registers, cache) that must be considered have limited access via OCD the proposed solution still offers a fast and logic efficient alternative. The migration of some features to the inside of the OCD allows better performance at the cost of little additional logic overhead on the target OCD circuitry. The standardization of OCD capabilities and access ports would also benefit the reusability of this fault injection approach.

Ongoing work is aimed at applying the proposed solutions to different target architectures and fault tolerant techniques. Simultaneously, means to further improve performance and coverage without incurring on unacceptable logic overhead and intrusiveness are also being studied.

\section{REFERENCES}

[1] "Coping with SEUs/SETs in microprocessors by means of low-cost solutions: A comparison study"; M. Rebaudengo,
M. S. Reorda, M. Violante, B. Nicolescu, R. Velazco; IEEE Transactions on Nuclear Science, Vol 49, No 3; June 2002.

[2] "Basic concepts and taxonomy of dependable and secure computing"; A. Avizienis, J.C. Laprie, B. Randell, C. Landwehr; IEEE Transactions on Dependable and Secure Computing, Volume 1, Issue 1; Jan 2004.

[3] "Comparison and application of different VHDL-based fault injection techniques”; J. Gracia, J.C. Baraza, D. Gil, P.J. Gil; IEEE International Symposium on Defect and Fault Tolerance in VLSI Systems; San Francisco, USA; Oct 2001.

[4] "Experimental evaluation of a COTS system for space applications"; H. Madeira, R. R. Some, F. Moreira, D. Costa, D. Rennels; International Conference on Dependable Systems and Networks; Bethesda, USA; June 2002.

[5] "Experimental Validation of High-Speed Fault-Tolerant Systems Using Physical Fault Injection"; R. J. Martínez, P. J. Gil, G. Martín, C. Pérez, J. J. Serrano; Seventh IFIP Working Conf. Dependable Computing for Critical Applications: DCCA-7; San Jose, USA; Jan. 1999.

[6] "Evaluation of the Thor Microprocessor Using Scan-chainBased and Simulation Based Fault-Injection"; P. Folkesson, S. Svensson, J. Karlsson; 8th European Workshop on Dependable Computing (EWDC-8); Goteborg, Sweden; April 1997.

[7] "A Technique for Automated Validation of Fault Tolerant Designs Using Laser Fault Injection (LFI)"; J. R. Samson, W. A. Moreno, F. J. Falquez; 28th Annual International Symposium on Fault-Tolerant Computing; Munich, Germany; June 1998.

[8] "Comparison of physical and software-implemented fault injection techniques"; J. Arlat, Y. Crouzet, J. Karlsson, P. Folkesson, E. Fuchs, G. H. Leber, IEEE Transactions on Computers, Volume 52, Issue 9; Sept. 2003.

[9] "The Nexus 5001 Forum Standard for a Global Embedded Processor Interface version 2.0”; IEEE-ISTO 5001; 2003.

[10] "INERTE: Integrated NExus-Based Real-Time Fault Injection Tool for Embedded Systems"; P. Yuste, D. de Andrés, L. Lemus, J. J. Serrano, P. J. Gil; The International Conference on Dependable Systems and Networks; San Francisco, USA; June 2003.

[11] www.freescale.com

[12] www.isystem.com/Products/Emulators/iC3000/

[13] Giovanni Ferrante, “CPUGEN 2.00”, 2003.

[14] www.opencores.org

[15] "How to characterize the problem of SEU in processors \& representative errors observed on flight"; R. Velazco, R. Ecoffet, F. Faure; 11th IEEE International On-Line Testing Symposium; Saint Raphael, France; July 2005.

[16] "A Modified Debugging Infrastructure to Assist Real Time Fault Injection Campaigns"; A. Fidalgo, G. Alves, J. Ferreira; 9th IEEE Workshop on Design and Diagnostics of Electronic Circuits and Systems (in press); Prague, Czech Republic; April 2006.

[17] www.xilinx.com 